\title{
Multiple Solutions for the Asymptotically Linear Kirchhoff Type Equations on $\mathbb{R}^{N}$
}

\author{
Yu Duan ${ }^{1,2}$ and Chun-Lei Tang \\ ${ }^{1}$ School of Mathematics and Statistics, Southwest University, Chongqing 400715, China \\ ${ }^{2}$ College of Science, Guizhou University of Engineering Science, Bijie, Guizhou 551700, China \\ Correspondence should be addressed to Chun-Lei Tang; tangcl@swu.edu.cn
}

Received 7 July 2016; Accepted 19 September 2016

Academic Editor: Wenming Zou

Copyright (C) 2016 Y. Duan and C.-L. Tang. This is an open access article distributed under the Creative Commons Attribution License, which permits unrestricted use, distribution, and reproduction in any medium, provided the original work is properly cited.

\begin{abstract}
The multiplicity of positive solutions for Kirchhoff type equations depending on a nonnegative parameter $\lambda$ on $\mathbb{R}^{N}$ is proved by using variational method. We will show that if the nonlinearities are asymptotically linear at infinity and $\lambda>0$ is sufficiently small, the Kirchhoff type equations have at least two positive solutions. For the perturbed problem, we give the result of existence of three positive solutions.
\end{abstract}

\section{Introduction and Main Results}

The purpose of this article is to investigate the multiplicity of positive solutions to the following nonlocal Kirchhoff type equations:

$$
\begin{aligned}
(a & \left.+\lambda\left(\int_{\mathbb{R}^{N}}\left(|\nabla u|^{2}+V(x) u^{2}\right) d x\right)\right)(-\Delta u+V(x) u) \\
& =q(x) f(u)+h(x) \text { in } \mathbb{R}^{N}
\end{aligned}
$$

where $N \geq 3, a$ is a positive constant, $\lambda>0$ is a parameter, and $f: \mathbb{R} \rightarrow \mathbb{R}$ is a continuous function.

In recent years, the following Kirchhoff type equation

$$
-\left(a+b \int_{\mathbb{R}^{N}}|\nabla u|^{2} d x\right) \Delta u+V(x) u=f(x, u)
$$

$$
\text { in } \mathbb{R}^{N} \text {, }
$$

has been studied by many researchers under variant assumptions on $V$ and $f$. Problem (2) is often referred to as nonlocal problem because of the appearance of the term $\left(\int_{\mathbb{R}^{N}}|\nabla u|^{2} d x\right) \Delta u$ which implies that (2) is no longer a pointwise identity. This causes some mathematical difficulties which make the study of (2) particularly interesting. Problem
(2) arises in an interesting physical context. Indeed, replacing $\mathbb{R}^{N}$ by a smooth bounded domain $\Omega \subset \mathbb{R}^{N}$ and setting $V(x)=0$, then problem (2) becomes the following Kirchhoff type Dirichlet problem:

$$
\begin{aligned}
-\left(a+b \int_{\Omega}|\nabla u|^{2} d x\right) \Delta u & =f(x, u), \quad \text { in } \Omega, \\
u & =0, \quad \text { on } \partial \Omega,
\end{aligned}
$$

which is related to the stationary analogue of the Kirchhoff equation

$$
u_{t t}-\left(a+b \int_{\Omega}|\nabla u|^{2} d x\right) \Delta u=f(x, u)
$$

that was presented by Kirchhoff [1] as a generalization of the well-known d'Alembert's equation

$$
\rho \frac{\partial^{2} u}{\partial t^{2}}-\left(\frac{P_{0}}{h}+\frac{E}{2 L} \int_{0}^{L}\left|\frac{\partial u}{\partial x}\right|^{2} d x\right) \frac{\partial^{2} u}{\partial x^{2}}=f(x, u)
$$

for free vibrations of elastic strings. Kirchhoff's model takes into account the changes in length of the string produced by transverse vibrations. The readers can learn some early classical research of Kirchhoff equations from [2,3]. However, 
(4) received great attention only after Lions [4] proposed an abstract framework to the problem. Some interesting results for problem (4) can be found in [5-7] and the references therein. There have been many works about the existence and multiplicity of nontrivial solutions to problem (3) using variational methods (see [8-18] and the references therein). Nevertheless, the problems they studied were based on a bounded domain of $\Omega \subset \mathbb{R}^{N}$. Very recently, some authors had studied the Kirchhoff type equation on the whole space $\mathbb{R}^{N}$. Many solvability conditions with $f$ near zero and infinity for problem (2) have been considered, such as the superlinear case (see [19-28]); the asymptotically linear case (see [29, 30]); the sublinear case (see [31-33]).

Particularly, the following Kirchhoff type problem has been studied widely by some authors under various conditions on $f$ and $V$ :

$$
\begin{aligned}
(a & \left.+\lambda\left(\int_{\mathbb{R}^{N}}\left(|\nabla u|^{2}+V(x) u^{2}\right) d x\right)\right)(-\Delta u+V(x) u) \\
& =f(x, u), \quad \text { in } \mathbb{R}^{N} .
\end{aligned}
$$

When $f(x, u)=|u|^{p-2} u, p \in\left(2,2^{*}\right)$, Huang and Liu [34] considered (6) and studied existence and nonexistence of positive solution by variational methods; they also discussed the energy doubling property of nodal solutions by Nehari manifold; Wu et al. [35] gave a total description on the positive solutions to (6), and they make an observation on the sign-changing solutions. The results of [34], respectively, complement the corresponding results of $[25,36] . \mathrm{Li}$ and Ye [25] showed that problem (6) has no nontrivial solution provided $f(x, u)=|u|^{p^{-2}} u, p \in(2,3)$ when $\lambda>0$ is sufficiently large. If $V(x)=b$, Liu et al. [37] studied the existence of a positive solution for problem (6) involving subcritical growth, which unifies and sharply improves the results of [36]. Fan and Liu [38] studied (6) with concaveconvex nonlinearities and showed that problem (6) has at least two positive solutions for $\lambda>0$ sufficiently small. When $f(x, u)$ is asymptotically linear with respect to $u$ at infinity, Ye and Yin [39] studied (6) and proved the existence of positive solution for $\lambda$ sufficiently small and the nonexistence result for $\lambda$ sufficiently large. When $V(x)=V(|x|), f(x, u)=f(u)$ is asymptotically linear with respect to $u$ at infinity; Li and Sun [40] showed the existence, nonexistence, and multiplicity to (6) in radial space $H_{r}^{1}\left(\mathbb{R}^{N}\right)$. When the nonlinearities $f$ is sublinear or local sublinear, $[41,42]$ considered the existence and multiplicity of nontrivial solutions to problem (6). Recently, some authors extend problem (6) to the $p$-Kirchhoff elliptic equations (see, e.g., [43-49] and the references therein). In all works for (6) mentioned above except for [39, 40], we found that the nonlinearities $f$ are superlinear, sublinear, or local sublinear. To the best of our knowledge, there is little information on the multiplicity of solution for (6) with the nonlinearities $f$ satisfying the asymptotically linear condition at infinity. In this paper, we will try to study multiplicity of positive solutions for problem (1) when $f$ is asymptotically linear at infinity.
In order to reduce our statements, we make the following assumptions:

$(V) V(x) \in C\left(\mathbb{R}^{N}, \mathbb{R}\right)$ satisfies $\inf _{\mathbb{R}^{N}} V(x) \geq V_{0}>0$, where $V_{0}$ is a constant.

$\left(F_{1}\right) f \in C\left(\mathbb{R}, \mathbb{R}^{+}\right), \lim _{t \rightarrow 0}(f(t) / t)=0$, and $f(t) \equiv 0$ for all $t \leq 0$.

$\left(F_{2}\right)$ There exists $l \in(0,+\infty)$ such that $\lim _{t \rightarrow \infty}(f(t) /$ $t)=l$.

$\left(F_{3}\right)$ There exists $\alpha \in(N / 2,+\infty)$ such that $q(x) \in$ $L^{\alpha}\left(\mathbb{R}^{N}\right)$.

$\left(F_{4}\right) l / a>\mu^{*}:=\inf \left\{\int_{\mathbb{R}^{N}}\left(|\nabla u|^{2}+V(x) u^{2}\right) d x: u \epsilon\right.$ $\left.H, \int_{\mathbb{R}^{N}} q(x) u^{2} d x=1\right\}$, where $H$ will be given below. $\left(F_{5}\right) 0 \leq h \in L^{2}\left(\mathbb{R}^{N}\right)$.

Before stating our main results, we give several notations. Set

$$
H^{1}\left(\mathbb{R}^{N}\right):=\left\{u \in L^{2}\left(\mathbb{R}^{N}\right):|\nabla u| \in L^{2}\left(\mathbb{R}^{N}\right)\right\}
$$

with the usual norm

$$
\|u\|_{H^{1}}=\left(\int_{\mathbb{R}^{N}}\left(|\nabla u|^{2}+u^{2}\right) d x\right)^{1 / 2} .
$$

Let

$$
\begin{aligned}
& H \\
& =\left\{u \in H^{1}\left(\mathbb{R}^{N}\right): \int_{\mathbb{R}^{N}}\left(|\nabla u|^{2}+V(x) u^{2}\right) d x<+\infty\right\}
\end{aligned}
$$

with the inner product and the norm

$$
\begin{aligned}
\langle u, v\rangle & =\int_{\mathbb{R}^{N}}(\nabla u \cdot \nabla v+V(x) u v) d x, \\
\|u\| & =\langle u, u\rangle^{1 / 2} .
\end{aligned}
$$

Since $V(x)$ satisfies $(V)$, it is easy to see that $\|\cdot\|_{H^{1}}$ is equivalent to $\|\cdot\|$. Obviously, the embedding $H \hookrightarrow L^{\mathcal{s}}\left(\mathbb{R}^{N}\right)$ is continuous for any $s \in\left[2,2^{*}\right]$. We denote by $\|\cdot\|_{p}$ the usual $L^{p}\left(\mathbb{R}^{N}\right)$ norm.

Define the functional $I_{\lambda}, J_{\lambda}: H \rightarrow \mathbb{R}$ by

$$
\begin{aligned}
& I_{\lambda}(u)=\frac{1}{2} a\|u\|^{2}+\frac{1}{4} \lambda\|u\|^{4}-\int_{\mathbb{R}^{N}} q(x) F(u) d x, \\
& J_{\lambda}(u)=I_{\lambda}(u)-\int_{\mathbb{R}^{N}} h(x) u^{+} d x,
\end{aligned}
$$

$u \in H$

where $F(t)=\int_{0}^{t} f(s) d s$. Clearly, by the assumptions imposed on $f, g$, and $h$, we know that $I_{\lambda}(u)$ and $J_{\lambda}(u)$ are well defined on $H$, and $I_{\lambda}, J_{\lambda} \in C^{1}(H, \mathbb{R})$ with the derivative given by

$$
\begin{aligned}
& \left\langle I_{\lambda}^{\prime}(u), v\right\rangle \\
& \quad=\left(a+\lambda\|u\|^{2}\right) \int_{\mathbb{R}^{N}}(\nabla u \cdot \nabla v+V(x) u v) d x
\end{aligned}
$$




$$
\begin{gathered}
-\int_{\mathbb{R}^{N}} q(x) f(u) v d x \\
\left\langle J_{\lambda}^{\prime}(u), v\right\rangle=\left\langle I_{\lambda}^{\prime}(u), v\right\rangle-\int_{\mathbb{R}^{N}} h(x) v^{+} d x,
\end{gathered}
$$$$
u, v \in H
$$

It is standard to verify that the weak solutions of (1) correspond to the critical points of the functional $J_{\lambda}$. Our first result for (1) without $h(x)$ is as follows.

Theorem 1. Assume that $N \geq 3$, $a$ is a positive constant, and $\lambda>0$ is a parameter. If the conditions $(V)$ and $\left(F_{1}\right)-\left(F_{4}\right)$ hold, then there exists $\lambda^{*}>0$ such that, for any $\lambda \in\left(0, \lambda^{*}\right)$, problem (1) has at least two positive solutions.

Remark 2. Compared with the works mentioned above except $[39,40]$, where the nonlinearities $f$ are superlinear, sublinear, or local sublinear, here we consider problem (1) with asymptotically linear nonlinearities. So, our problem is different and extend the abovementioned results to some extent.

Remark 3. In [39], the authors only studied the existence of positive solutions. In this paper, we give multiplicity results when the potential $V$ is different from the conditions of $V$ in [39] and our method is simpler than that used in [39]. When $V(x)=V(|x|)$ satisfied some assumptions, Li and Sun [40] showed the existence, nonexistence, and multiplicity of radial solutions. Here, we get multiplicity results in nonradial space.

Remark 4. Indeed, it is not difficult to find some functions $f(t), V(x)$, and $q(x)$ such that the conditions of Theorem 1 are satisfied. For example, for any fixed $R_{0}>0$, let

$$
\begin{aligned}
& f(t)= \begin{cases}\frac{R_{0} t^{2}}{1+t}, & t>0, \\
0, & t \leq 0,\end{cases} \\
& q(x)= \begin{cases}\frac{1}{1+|x|}, & 0 \leq|x| \leq R_{0}, \\
\epsilon L^{\alpha}\left(B_{R_{0}}^{c}(0)\right), & |x| \geq R_{0} .\end{cases}
\end{aligned}
$$

Choosing $V(x)=1$, it is easy to know that $(V)$ and $\left(F_{1}\right)-\left(F_{3}\right)$ are satisfied for any $R_{0}>0$. Moreover, $l=R_{0}$ in $\left(F_{2}\right)$ and $F(t)=R_{0}\left(\left(t^{2}-2 t\right) / 2+\ln (1+t)\right)$. To verify the condition $\left(F_{4}\right)$, we have to choose a special $R_{0}>0$. Indeed, for $R>0$, take $\psi \in C_{0}^{\infty}\left(\mathbb{R}^{N},[0,1]\right)$ such that

$$
\psi(x)= \begin{cases}1, & |x| \leq R \\ 0, & |x| \geq 2 R\end{cases}
$$

and $|\nabla \psi(x)| \leq C / R$ for all $x \in \mathbb{R}^{N}$, where $C>0$ is a constant independent of $x$. Because of supp $\psi \subset B_{2 R}$, thus for $R_{0}>2 R$, we have

$$
\begin{aligned}
\frac{\int_{\mathbb{R}^{N}}\left(|\nabla \psi|^{2}+V(x) \psi^{2}\right) d x}{\int_{\mathbb{R}^{N}} q(x) \psi^{2} d x} \\
=\frac{\int_{|x| \leq 2 R}\left(|\nabla \psi|^{2}+V(x) \psi^{2}\right) d x}{\int_{|x| \leq R} q(x) \psi^{2} d x}
\end{aligned}
$$

$$
\begin{aligned}
& \leq \frac{\int_{|x| \leq 2 R}\left(1+C^{2} / R^{2}\right) d x}{\int_{|x| \leq R}(1 /(1+|x|)) d x} \\
& \leq C^{\prime}(R+1)+\frac{(R+1) C^{\prime} C^{2}}{R^{2}},
\end{aligned}
$$

where $C^{\prime}$ is a constant independent of $R$. So, choosing $R$ sufficiently large such that $(R+1) C^{2} / R^{2} \leq 1$, the condition $\left(F_{4}\right)$ holds for $R_{0}=a C^{\prime}(R+3)$.

In our second result, we consider the case of the perturbed Kirchhoff equations; that is, $h(x) \neq 0$, and we obtain the following result.

Theorem 5. Assume that $N \geq 3$, $a$ is a positive constant, and $\lambda>0$ is a parameter. If the conditions $(V)$ and $\left(F_{1}-F_{5}\right)$ hold, then there exist two constants $\tilde{\lambda}>0$ and $m_{0}>0$ such that, for any $\lambda \in(0, \tilde{\lambda})$, problem (1) has at least three positive solutions when $\|h\|_{2}<m_{0}$.

Remark 6. In the aforementioned papers, the nonlinearities satisfy $f(x, 0)=0$. Indeed, this condition is not necessary. Here, the nonlinearity may not be 0 at zero because of $f(x, 0)=h(x) \geq 0$.

In order to obtain our results, we have to overcome various difficulties. On the one hand, it is well known that Sobolev embedding $H \hookrightarrow L^{p}\left(\mathbb{R}^{N}\right)$ is continuous but not compact for $p \in\left[2,2^{*}\right)$, and then it is usually difficult to prove that a minimizing sequence or a Palais-Smale sequence is strongly convergent if we seek solutions of problem (1) by variational methods. To overcome this difficulty, we make full use of integrability of potential function $q(x)$ and perturbation $h(x)$. On the other hand, as we all know, the (PS) sequence is bounded if the nonlinearity satisfies a variant of Ambrosetti-Rabinowitz type condition ((AR) in short) or 4-superlinearity. However, for the asymptotically linear case of problem (1), we can adopt a simple method to verify the boundedness of (PS) sequence. The conditions $\left(F_{1}\right)$ and $\left(F_{2}\right)$ are crucial to obtain the boundedness of (PS) sequence.

This paper is organized as follows. We give some previous results and prove Theorem 1 in Section 2. Section 3 is devoted to giving the proof of Theorem 5 . Throughout this paper, $C$ and $C_{i}$ are used in various places to denote distinct constants.

\section{Proof of Theorem 1}

In the following, we give some lemmas which are important to prove our main result.

Lemma 7. Suppose that $(V)$ and $\left(F_{1}\right)-\left(F_{3}\right)$ hold; then, $I_{\lambda}(u)$ is coercive on $H$.

Proof. By $\left(F_{1}\right)$ and $\left(F_{2}\right)$, we see that $f(s) / s$ is bounded in $\mathbb{R}$. So, setting $L_{0}=\sup _{s \in \mathbb{R}}(f(s) / s)$, thus $L_{0} \in(0,+\infty)$ and for any $s \in \mathbb{R}$

$$
0 \leq \frac{f(s)}{s} \leq L_{0}
$$


Then,

$$
|F(s)| \leq \frac{L_{0}}{2}|s|^{2}, \quad \forall s \in \mathbb{R} .
$$

Because of $\alpha \in(N / 2,+\infty)$, we have

$$
2<\frac{2 \alpha}{\alpha-1}<2^{*}
$$

Furthermore, by (17), (18), $\left(F_{3}\right)$, and the Hölder and Sobolev inequalities, we deduce that for any $u \in H$

$$
\begin{aligned}
& \left|\int_{\mathbb{R}^{N}} q(x) F(u) d x\right| \leq \frac{L_{0}}{2} \int_{\mathbb{R}^{N}} q(x) u^{2} d x \\
& \quad \leq \frac{L_{0}}{2}\left(\int_{\mathbb{R}^{N}} q^{\alpha}(x) d x\right)^{1 / \alpha}\left(\int_{\mathbb{R}^{N}} u^{2 \alpha /(\alpha-1)} d x\right)^{(\alpha-1) / \alpha} \\
& \quad \leq \frac{L_{0}}{2}\left(\int_{\mathbb{R}^{N}} q^{\alpha}(x) d x\right)^{1 / \alpha}\|u\|_{2 \alpha /(\alpha-1)}^{2} \\
& \quad \leq \frac{C_{1} L_{0}}{2}\|u\|^{2} .
\end{aligned}
$$

Thus, we obtain

$$
\begin{aligned}
I_{\lambda}(u) & =\frac{1}{2} a\|u\|^{2}+\frac{1}{4} \lambda\|u\|^{4}-\int_{\mathbb{R}^{N}} q(x) F(u) d x \\
& \geq \frac{1}{2} a\|u\|^{2}+\frac{1}{4} \lambda\|u\|^{4}-\frac{C_{1} L_{0}}{2}\|u\|^{2},
\end{aligned}
$$

which shows that $I_{\lambda}(u)$ is coercive on $H$.

It follows from Lemma 7 that $I_{\lambda}$ is bounded from below on $H$ and thus we may define $c_{\lambda}:=\inf _{H} I_{\lambda}$.

Lemma 8. Assume that $(V)$ and $\left(F_{1}-F_{4}\right)$ are satisfied; then, $I_{\lambda}(u)$ satisfies the $(P S)$ condition.

Proof. Suppose that $\left\{u_{n}\right\} \subset H$ is the (PS) sequence for the functional $I_{\lambda}$; that is,

$$
\begin{gathered}
\left\{I_{\lambda}\left(u_{n}\right)\right\} \text { is bounded, } \\
I_{\lambda}^{\prime}\left(u_{n}\right) \longrightarrow 0 \text { as } n \longrightarrow \infty .
\end{gathered}
$$

By Lemma 7 , the sequence $\left\{u_{n}\right\}$ is bounded in $H$. Going if necessary to a subsequence, we can assume that $u_{n} \rightarrow u$ weakly in $H$ for some $u \in H$. Now, we begin to prove $u_{n} \rightarrow u$ strongly in $H$. As we all know, it is sufficient to show that $\left\|u_{n}\right\| \rightarrow\|u\|$ as $n \rightarrow \infty$. By (21), we see that

$$
\begin{aligned}
o(1)= & \left\langle I_{\lambda}^{\prime}\left(u_{n}\right), u_{n}-u\right\rangle \\
= & \left(a+\lambda\left\|u_{n}\right\|^{2}\right)\left\langle u_{n}, u_{n}-u\right\rangle \\
& -\int_{\mathbb{R}^{N}} q(x) f\left(u_{n}\right)\left(u_{n}-u\right) d x .
\end{aligned}
$$

So, we have

$$
\begin{aligned}
(a & \left.+\lambda\left\|u_{n}\right\|^{2}\right)\left\langle u_{n}, u_{n}-u\right\rangle \\
& =o(1)+\int_{\mathbb{R}^{N}} q(x) f\left(u_{n}\right)\left(u_{n}-u\right) d x .
\end{aligned}
$$

Thus, to show that $\left\langle u_{n}, u_{n}-u\right\rangle=o(1)$ is equivalent to proving that

$$
\int_{\mathbb{R}^{N}} q(x) f\left(u_{n}\right)\left(u_{n}-u\right) d x=o(1) .
$$

By $\left(F_{3}\right)$, for any $\varepsilon>0$, there exists $R_{\varepsilon}>0$ such that

$$
\int_{|x| \geq R} q^{\alpha}(x) d x<\varepsilon^{\alpha}, \quad \forall R \geq R_{\varepsilon} .
$$

By (16), (18), (25), $\left(F_{3}\right)$, and the Sobolev and Hölder inequalities, we have

$$
\begin{aligned}
& \left|\int_{\mathbb{R}^{N}} q(x) f\left(u_{n}\right)\left(u_{n}-u\right) d x\right| \\
& \quad \leq \int_{\mathbb{R}^{N}}\left|q(x)\left(u_{n}-u\right)\right|\left|f\left(u_{n}\right)\right| d x \\
& \quad \leq L_{0} \int_{|x| \leq R}\left|q(x)\left(u_{n}-u\right)\right|\left|u_{n}\right| d x \\
& \quad+L_{0} \int_{|x| \geq R}\left|q(x)\left(u_{n}-u\right)\right|\left|u_{n}\right| d x \\
& \quad \leq L_{0}\|q\|_{L^{\alpha}\left(B_{R}(0)\right)}\left\|u_{n}\right\|_{L^{2 \alpha /(\alpha-1)}\left(B_{R}(0)\right)} \\
& \quad \cdot\left\|u_{n}-u\right\|_{L^{2 \alpha /(\alpha-1)}\left(B_{R}(0)\right)}+L_{0}\|q\|_{L^{\alpha}\left(B_{R}^{c}(0)\right)} \\
& \quad \cdot\left\|u_{n}-u\right\|_{L^{2 \alpha /(\alpha-1)}\left(B_{R}^{c}(0)\right)}\left\|u_{n}\right\|_{L^{2 \alpha /(\alpha-1)}\left(B_{R}^{c}(0)\right)} \leq o(1) \\
& \quad+C_{2}\|q\|_{L^{\alpha}\left(B_{R}^{c}(0)\right)}\left\|u_{n}-u\right\|\left\|u_{n}\right\| \leq o(1)+C_{3} \varepsilon
\end{aligned}
$$

This implies

$$
\int_{\mathbb{R}^{N}} q(x) f\left(u_{n}\right)\left(u_{n}-u\right) d x=o(1) .
$$

So, $\left\langle u_{n}, u_{n}-u\right\rangle \rightarrow 0$. It is easy to see that $\left\langle u, u_{n}-u\right\rangle \rightarrow 0$. Hence, $\left\langle u_{n}-u, u_{n}-u\right\rangle \rightarrow 0$; that is, $u_{n} \rightarrow u$ strongly in $H$.

Proof of Theorem 1. The proof of this theorem is divided into two steps.

Step 1. In this step, we will show that problem (1) has a mountain pass solution.

By $\left(F_{3}\right)$, we see that $\alpha>N / 2$, and thus $2^{*}(\alpha-1) / \alpha>2$. So, we may choose a constant $\beta \in\left(2,2^{*}(\alpha-1) / \alpha\right]$ such that $\beta \alpha /(\alpha-1) \leq 2^{*}$. For any $\varepsilon>0$, it follows from $\left(F_{1}\right)$ and $\left(F_{2}\right)$ that there exists $C_{\varepsilon}>0$ and $\beta \in\left(2,2^{*}(\alpha-1) / \alpha\right]$ such that

$$
|f(s)| \leq \varepsilon|s|+C_{\varepsilon}|s|^{\beta-1}, \quad \forall s \in \mathbb{R}
$$

and, then,

$$
|F(s)| \leq \frac{\varepsilon}{2}|s|^{2}+\frac{C_{\varepsilon}}{\beta}|s|^{\beta}, \quad \forall s \in \mathbb{R} .
$$


Using (29), $\left(F_{3}\right)$, and Sobolev and Hölder inequalities, we deduce that for any $u \in H$

$$
\begin{aligned}
& \left|\int_{\mathbb{R}^{N}} q(x) F(u) d x\right| \leq \frac{\varepsilon}{2} \int_{\mathbb{R}^{N}} q(x) u^{2} d x \\
& +\frac{C_{\varepsilon}}{\beta} \int_{\mathbb{R}^{N}} q(x)|u|^{\beta} d x \\
& \quad \leq \frac{\varepsilon}{2}\left(\int_{\mathbb{R}^{N}} q^{\alpha}(x) d x\right)^{1 / \alpha}\left(\int_{\mathbb{R}^{N}} u^{2 \alpha /(\alpha-1)} d x\right)^{(\alpha-1) / \alpha} \\
& +\frac{C_{\varepsilon}}{\beta}\left(\int_{\mathbb{R}^{N}} q^{\alpha}(x) d x\right)^{1 / \alpha}\left(\int_{\mathbb{R}^{N}} u^{\beta \alpha /(\alpha-1)} d x\right)^{(\alpha-1) / \alpha} \\
& \quad \leq \frac{\varepsilon}{2}\left(\int_{\mathbb{R}^{N}} q^{\alpha}(x) d x\right)^{1 / \alpha}\|u\|_{2 \alpha /(\alpha-1)}^{2} \\
& +\frac{C_{\varepsilon}}{\beta}\left(\int_{\mathbb{R}^{N}} q^{\alpha}(x) d x\right)^{1 / \alpha}\|u\|_{\beta \alpha /(\alpha-1)}^{\beta} \leq \frac{C_{4} \varepsilon}{2}\|u\|^{2} \\
& +\frac{C_{5} C_{\varepsilon}\|u\|^{\beta}}{\beta} .
\end{aligned}
$$

Thus, we obtain

$$
\begin{aligned}
I_{\lambda}(u) & =\frac{1}{2} a\|u\|^{2}+\frac{1}{4} \lambda\|u\|^{4}-\int_{\mathbb{R}^{N}} q(x) F(u) d x \\
& \geq \frac{a-C_{4} \varepsilon}{2}\|u\|^{2}-\frac{C_{5} C_{\varepsilon}}{\beta}\|u\|^{\beta} .
\end{aligned}
$$

So, fixing $\varepsilon \in\left(0, a / C_{4}\right)$ and letting $\|u\|=\rho>0$ sufficiently small, it is easy to see that there exists a constant $\alpha>0$ such that

$$
\left.I_{\lambda}(u)\right|_{\|u\|=\rho} \geq \alpha>0 .
$$

By $\left(F_{4}\right)$, there is $v \in H$ such that $v \geq 0, \int_{\mathbb{R}^{N}} q(x) v^{2} d x=1$, and $\mu^{*} \leq\|v\|^{2}<l / a$. Combining $\left(F_{2}\right)$ with Fatou's lemma, we deduce that

$$
\begin{aligned}
\lim _{t \rightarrow+\infty} \frac{I_{0}(t v)}{t^{2}} & =\frac{a}{2}\|v\|^{2}-\lim _{t \rightarrow \infty} \int_{\mathbb{R}^{N}} q(x) \frac{F(t v)}{t^{2}} d x \\
& \leq \frac{1}{2}\left(a\|v\|^{2}-l\right)<0,
\end{aligned}
$$

which implies that there exist $e \in H$ with $\|e\|>\rho$ such that $I_{0}(e)<0$. Since $I_{\lambda}(e) \rightarrow I_{0}(e)$ as $\lambda \rightarrow 0^{+}$, we see that there exists $\lambda^{*}>0$ such that $I_{\lambda^{*}}(e)<0$, and then

$$
I_{\lambda}(e)<I_{\lambda^{*}}(e)<0
$$

for all $\lambda \in\left(0, \lambda^{*}\right)$. From (32), (34), and Mountain Pass Theorem, there is a sequence $\left\{u_{n}\right\} \subset H$ such that

$$
\begin{gathered}
\left\{I_{\lambda}\left(u_{n}\right)\right\} \text { is bounded, } \\
I_{\lambda}^{\prime}\left(u_{n}\right) \longrightarrow 0, \text { as } n \longrightarrow \infty .
\end{gathered}
$$

Using Lemma 8, we know $I_{\lambda}$ satisfies (PS)-condition. So, by Theorem 2.2 in [50], $I_{\lambda}$ possess a critical point $v_{0}$ with $I_{\lambda}\left(v_{0}\right) \geq \alpha>0$. Setting $v^{-}=\max \{0,-v\}$, since $(a+$ $\left.\lambda\left\|v_{0}\right\|^{2}\right)\left\langle v_{0}, v_{0}^{-}\right\rangle-\int_{\mathbb{R}^{N}} q(x) f\left(v_{0}\right) v_{0}^{-} d x=0$, then by $\left(F_{1}\right)$ we have

$$
\left\|v_{0}^{-}\right\|=0
$$

which implies $v_{0} \geq 0$ a.e. in $\mathbb{R}^{N}$. By the strong maximum principle, $v_{0}$ is positive on $H$ and $I_{\lambda}\left(v_{0}\right)>0$.

Step 2. Problem (1) has a global minimum; that is, there exists a positive function $u_{0} \in H$ such that $I_{\lambda}^{\prime}\left(u_{0}\right)=0$ and $c_{\lambda}=$ $\inf _{H} I_{\lambda}=I_{\lambda}\left(u_{0}\right)<0$.

From Lemmas 7 and 8, we know that $I_{\lambda}(u)$ is bounded from below and satisfies (PS)-condition, and then by Theorem 4.4 in [51], $c_{\lambda}=\inf _{H} I_{\lambda}$ is a critical value of $I_{\lambda}$; that is, there exists a function $u_{0} \in H$ such that $I_{\lambda}^{\prime}\left(u_{0}\right)=0$ and $I_{\lambda}\left(u_{0}\right)=c_{\lambda}$. In view of $(34)$, we know $c_{\lambda}=I_{\lambda}\left(u_{0}\right) \leq I_{\lambda}(e)<0$, which implies that $u_{0} \neq 0$, and using the same arguments as in Step 1, it is easy to know that $u_{0}$ is positive.

Because of $I_{\lambda}\left(v_{0}\right)>0>I_{\lambda}\left(u_{0}\right)$, we get two different critical points $v_{0}, u_{0}>0$; that is, problem (1) has two positive solutions, and then the proof of Theorem 1 is completed.

\section{Proof of Theorem 5}

First, we need the following lemmas which are important to prove Theorem 5 .

Lemma 9. Suppose that $(V)$ and $\left(F_{1}\right)-\left(F_{5}\right)$ hold; then, $J_{\lambda}(u)$ is coercive on $H$.

Proof. The proof is similar to the proof of Lemma 7, so we omit it here.

Lemma 10. Assume that $(V)$ and $\left(F_{1}\right)-\left(F_{5}\right)$ are satisfied; then, $J_{\lambda}(u)$ satisfies the $(P S)$ condition.

Proof. By Lemma 8, we only need to show $\int_{\mathbb{R}^{N}} h(x)\left(u_{n}-\right.$ $u) d x=o(1)$. By $\left(F_{5}\right)$, for the above-given $\varepsilon>0$, there exists $R_{\varepsilon}^{\prime}>0$ such that

$$
\int_{|x| \geq R} h^{2}(x) d x<\varepsilon^{2}, \quad \forall R \geq R_{\varepsilon}^{\prime} .
$$

By (37) and the Sobolev and Hölder inequalities, we have

$$
\begin{aligned}
& \left|\int_{\mathbb{R}^{N}} h(x)\left(u_{n}-u\right) d x\right| \\
& \quad \leq \int_{|x| \leq R}\left|h(x)\left(u_{n}-u\right)\right| d x
\end{aligned}
$$




$$
\begin{aligned}
&+\int_{|x| \geq R}\left|h(x)\left(u_{n}-u\right)\right| d x \\
& \leq\|h\|_{L^{2}\left(B_{R}(0)\right)}\left\|u_{n}-u\right\|_{L^{2}\left(B_{R}(0)\right)} \\
& \quad+\|h\|_{L^{2}\left(B_{R}^{c}(0)\right)}\left\|u_{n}-u\right\|_{L^{2}\left(B_{R}^{c}(0)\right)} \leq o(1)+C^{\prime} \varepsilon ;
\end{aligned}
$$

that is

$$
\int_{\mathbb{R}^{N}} h(x)\left(u_{n}-u\right) d x=o(1) .
$$

Using (27) and (39), we obtain

$$
\begin{aligned}
& \int_{\mathbb{R}^{N}} q(x) f\left(u_{n}\right)\left(u_{n}-u\right) d x+\int_{\mathbb{R}^{N}} h(x)\left(u_{n}-u\right) d x \\
& =o(1) .
\end{aligned}
$$

So, $\left\langle u_{n}, u_{n}-u\right\rangle \rightarrow 0$. It is easy to see that $\left\langle u, u_{n}-u\right\rangle \rightarrow 0$. Hence, $\left\langle u_{n}-u, u_{n}-u\right\rangle \rightarrow 0$; that is, $u_{n} \rightarrow u$ strongly in $H$.

Proof of Theorem 5. The proof of this theorem is divided into four steps.

Step 1. In this step, we will show that problem (1) has a positive mountain pass solution.

Set

$$
I_{\lambda^{*}}(u)=\frac{1}{2} a\|u\|^{2}+\frac{1}{4} \lambda^{*}\|u\|^{4}-\int_{\mathbb{R}^{N}} q(x) F(u) d x,
$$

where $\lambda^{*}$ is given in Theorem 1. By Lemma 7, we known that $I_{\lambda^{*}}(u)$ is coercive on $H$. So we can define

$$
c_{\lambda^{*}}:=\inf _{H} I_{\lambda^{*}}
$$

Using (34), we have $c_{\lambda} \leq c_{\lambda^{*}}<0$. By (31), we know

$$
\begin{aligned}
J_{\lambda}(u) & \\
= & \frac{1}{2} a\|u\|^{2}+\frac{1}{4} \lambda\|u\|^{4}-\int_{\mathbb{R}^{N}} q(x) F(u) d x \\
& -\int_{\mathbb{R}^{N}} h(x) u^{+} d x \\
\geq & \frac{a-C_{4} \varepsilon}{2}\|u\|^{2}-\frac{C_{5} C_{\varepsilon}}{\beta}\|u\|^{\beta}-\frac{1}{\sqrt{V_{0}}}\|h\|_{2}\|u\| \\
\geq & \|u\|\left(\frac{a-C_{4} \varepsilon}{2}\|u\|-\frac{C_{5} C_{\varepsilon}}{\beta}\|u\|^{\beta-1}-\frac{1}{\sqrt{V_{0}}}\|h\|_{2}\right) .
\end{aligned}
$$

So, choosing $\varepsilon=a / 2 C_{4}$ and setting

$$
g(t)=\frac{a}{4} t-\frac{C_{5} C_{\varepsilon}}{\beta} t^{\beta-1}
$$

for $t \geq 0$, we see that there exists a constant $\rho_{1}>0$ sufficiently small such that $0<\rho_{1}<\delta:=\min \left\{\rho, \sqrt{\left(\sqrt{a^{2}-\lambda^{*} c_{\lambda}^{*}}-a\right) / \lambda^{*}}\right\}$ and $g\left(\rho_{1}\right)>0$, where $\beta \in\left(2,2^{*}(\alpha-1) / \alpha\right]$ and $\rho$ is given by (32). Taking $m_{1}:=\left(\sqrt{V_{0}} / 2\right) g\left(\rho_{1}\right)$, it then follows that there exists a constant $\alpha_{1}:=(1 / 2) g\left(\rho_{1}\right) \rho_{1}>0$ such that

$$
\left.J_{\lambda}(u)\right|_{\|u\|=\rho_{1}} \geq \alpha_{1}
$$

for all $h$ satisfying $\|h\|_{2}<m_{1}$.

Using the similar proof of (34), we can obtain that there exists a constant $\tilde{\lambda}>0$ and a function $e \in H$ with $\|e\|>\rho_{1}$ such that

$$
J_{\lambda}(e)<0
$$

for all $\lambda \in(0, \tilde{\lambda})$.

From (45), (46), and Mountain Pass Theorem, there is a sequence $\left\{u_{n}\right\} \subset H$ such that

$$
\begin{gathered}
\left\{J_{\lambda}\left(u_{n}\right)\right\} \text { is bounded, } \\
J_{\lambda}^{\prime}\left(u_{n}\right) \longrightarrow 0 \text { as } n \longrightarrow \infty .
\end{gathered}
$$

It follows from Lemma 10 that $J_{\lambda}$ satisfies (PS)-condition. So, using Theorem 2.2 in [50], $J_{\lambda}$ possess a critical point $u_{1}$ with $J_{\lambda}\left(u_{1}\right) \geq \alpha>0$ when $\|h\|_{2}<m_{1}$. Let $u^{-}=$ $\max \{0,-u\}$. Since $\left(a+\lambda\left\|u_{1}\right\|^{2}\right)\left\langle u_{1}, u_{1}^{-}\right\rangle-\int_{\mathbb{R}^{N}} q(x) f\left(u_{1}\right) u_{1}^{-} d x-$ $\int_{\mathbb{R}^{N}} h(x)\left(u_{1}^{-}\right)^{+} d x=0$, then by $\left(F_{1}\right)$ and $\left(F_{5}\right)$ we have

$$
\left\|u_{1}^{-}\right\|=0
$$

which implies $u_{1} \geq 0$ a.e. in $\mathbb{R}^{N}$. So, by the strong maximum principle, $u_{1}$ is positive on $H$.

Step 2. In this step, we prove the existence of local minimum solution for problem (1).

Since $h \in L^{2}\left(\mathbb{R}^{N}\right)$ and $h \geq 0$, we can choose a function $\psi \in H$ such that

$$
\int_{\mathbb{R}^{N}} h(x) \psi^{+} d x>0 .
$$

Hence, we obtain

$$
\begin{aligned}
\lim _{t \rightarrow 0} \frac{J_{\lambda}(t \psi)}{t} & =\lim _{t \rightarrow 0} \frac{\left(t^{2} / 2\right) a\|\psi\|^{2}+\left(t^{4} / 4\right) \lambda\|\psi\|^{4}-\int_{\mathbb{R}^{N}} q(x) F(t \psi) d x-\int_{\mathbb{R}^{N}} h(x) t \psi^{+} d x}{t} \\
& \leq \lim _{t \rightarrow 0}\left(\frac{t}{2} a\|\psi\|^{2}+\frac{t^{3}}{4} \lambda\|\psi\|^{4}-\int_{\mathbb{R}^{N}} h(x) \psi^{+} d x\right)=-\int_{\mathbb{R}^{N}} h(x) \psi^{+} d x<0 .
\end{aligned}
$$


Thus, we have

$$
c_{1}:=\inf \left\{J_{\lambda}(u): u \in \bar{B}_{\rho_{1}}\right\}<0,
$$

where $\rho_{1}$ is given by (45) and $B_{\rho_{1}}=\left\{u \in H:\|u\|<\rho_{1}\right\}$. By Ekeland's variational principle, there exists a sequence $\left\{u_{n}\right\} \subset$ $\bar{B}_{\rho}$ such that

$$
\begin{gathered}
c_{1} \leq J_{\lambda}\left(u_{n}\right)<c_{1}+\frac{1}{n}, \\
J_{\lambda}(v) \geq J_{\lambda}\left(u_{n}\right)-\frac{1}{n}\left\|v-u_{n}\right\|
\end{gathered}
$$

for all $v \in \bar{B}_{\rho_{1}}$. Then, by a standard procedure, we can show that $\left\{u_{n}\right\}$ is a bounded Palais-Smale sequence of $J_{\lambda}$. Therefore, Lemma 10 implies that there exists a function $u_{2} \in B_{\rho_{1}}$ such that $J_{\lambda}\left(u_{2}\right)=c_{1}<0$ and $J_{\lambda}^{\prime}\left(u_{2}\right)=0$. Similarly, $u_{2}>0$.

Step 3. Problem (1) has a global minimum.

It follows from Lemmas 9 and 10 that $J_{\lambda}(u)$ is bounded from below and satisfies the (PS) condition, so we may define $c_{2}:=\inf _{H} J_{\lambda}$. Using Theorem 4.4 in [51], $c_{2}=\inf _{H} J_{\lambda}$ is a critical value of $J_{\lambda}$; that is, there exists a critical point $u_{3} \in H$ such that $J_{\lambda}^{\prime}\left(u_{3}\right)=0$ and $J_{\lambda}\left(u_{3}\right)=c_{2}$. By (46), $c_{2}=J_{\lambda}\left(u_{3}\right)=$ $\inf _{H} J_{\lambda}<0$, which implies $u_{3} \neq 0$. Similarly, $u_{3}>0$.

Step 4. $u_{1}, u_{2}$, and $u_{3}$ are different from each other; that is, problem (1) has three positive solutions.

Since $J_{\lambda}\left(u_{1}\right)>0>J_{\lambda}\left(u_{2}\right)$ and $J_{\lambda}\left(u_{1}\right)>0>J_{\lambda}\left(u_{3}\right)$, thus $u_{1} \neq u_{2}$ and $u_{1} \neq u_{3}$. Next, we claim that $u_{2} \neq \equiv u_{3}$ and then the proof of Theorem 5 is completed. Using the proof of Theorem 1 , we know $I_{\lambda}\left(u_{0}\right)=c_{\lambda}<0$ and $I_{\lambda}(0)=0$. Using (31), $I_{\lambda}(u)>0$ for all $\|u\|<\rho$. Then,

$$
\begin{aligned}
\left|I_{\lambda}(u)\right| & =\left|\frac{1}{2} a\|u\|^{2}+\frac{1}{4} \lambda\|u\|^{4}-\int_{\mathbb{R}^{N}} q(x) F(u) d x\right| \\
& =\frac{1}{2} a\|u\|^{2}+\frac{1}{4} \lambda\|u\|^{4}-\int_{\mathbb{R}^{N}} q(x) F(u) d x \\
& \leq \frac{1}{2} a\|u\|^{2}+\frac{1}{4} \lambda^{*}\|u\|^{4}<-\frac{1}{4} c_{\lambda^{*}}
\end{aligned}
$$

uniformly in $\lambda$ when $\|u\|<\delta \quad:=\min \{\rho$, $\sqrt{\left(\sqrt{a^{2}-\lambda^{*} c_{\lambda}^{*}}-a\right) / \lambda^{*}}$. So, when $\|u\|<\delta$ and $\|h\|_{2}<m_{2}:=$ $-\sqrt{V_{0}} c_{\lambda^{*}} / 4 \delta$, we deduce that

$$
\begin{aligned}
J_{\lambda}(u) & =I_{\lambda}(u)-\int_{\mathbb{R}^{N}} h(x) u^{+} d x \\
& \geq \frac{1}{4} c_{\lambda^{*}}-\frac{1}{\sqrt{V_{0}}}\|h\|_{2}\|u\| \geq \frac{1}{4} c_{\lambda^{*}}-\frac{1}{\sqrt{V_{0}}}\|h\|_{2} \delta \\
& >\frac{1}{2} c_{\lambda^{*}} .
\end{aligned}
$$

Since $\rho_{1}<\delta$, using (54), we obtain

$$
\begin{aligned}
c_{1} & =\inf \left\{J_{\lambda}(u): u \in \bar{B}_{\rho_{1}}\right\}=J_{\lambda}\left(u_{2}\right)>\frac{1}{2} c_{\lambda^{*}}, \\
J_{\lambda}\left(u_{0}\right) & =I_{\lambda}\left(u_{0}\right)-\int_{\mathbb{R}^{N}} h(x) u_{0}^{+} d x \leq I_{\lambda}\left(u_{0}\right)=c_{\lambda} \\
& \leq c_{\lambda^{*}},
\end{aligned}
$$

when $\|h\|_{2}<m_{2}$. Thus, we have

$$
J_{\lambda}\left(u_{0}\right) \leq c_{\lambda^{*}}<\frac{1}{2} c_{\lambda^{*}}<J_{\lambda}\left(u_{2}\right) .
$$

So, $c_{2}=J_{\lambda}\left(u_{3}\right)=\inf _{H} J_{\lambda} \leq J_{\lambda}\left(u_{0}\right)<J_{\lambda}\left(u_{2}\right)$; that is, $u_{2} \neq \equiv$ $u_{3}$. Set $m_{0}:=\min \left\{m_{1}, m_{2}\right\}$. From the discussion above, we can obtain that problem (1) has three positive solutions when $\|h\|_{2}<m_{0}$.

\section{Competing Interests}

The authors declare that they have no competing interests.

\section{Acknowledgments}

The authors wish to express their gratitude to the referees for valuable comments and suggestions on the manuscript. This paper is supported by National Natural Science Foundation of China (nos. 11471267, 11361003) and the Science and Technology Foundation of Guizhou Province (nos. LH[2015]7595, LH[2016]7054, LKB[2013]24, LH[2014]7535).

\section{References}

[1] G. Kirchhoff, Mechanik, Teubner, Leipzig, Germany, 1883.

[2] S. Bernstein, "Sur une classe d'équations fonctionnelles aux dérivées partielles," Izvestiâ Akademii Nauk SSSR, vol. 4, no. 1, pp. 17-26, 1940.

[3] S. I. Pohožaev, "A certain class of quasilinear hyperbolic equations," Matematicheskii Sbornik, vol. 96, no. 138, pp. 152-168, 1975.

[4] J. L. Lions, "On some questions in boundary value problems of mathematical physics," North-Holland Mathematics Studies, vol. 30, pp. 284-346, 1978.

[5] A. Arosio and S. Panizzi, "On the well-posedness of the Kirchhoff string," Transactions of the American Mathematical Society, vol. 348, no. 1, pp. 305-330, 1996.

[6] M. M. Cavalcanti, V. N. Domingos Cavalcanti, and J. A. Soriano, "Global existence and uniform decay rates for the KirchhoffCarrier equation with nonlinear dissipation," Advances in Differential Equations, vol. 6, no. 6, pp. 701-730, 2001.

[7] P. D'Ancona and S. Spagnolo, "Global solvability for the degenerate Kirchhoff equation with real analytic data," Inventiones Mathematicae, vol. 108, no. 2, pp. 247-262, 1992.

[8] C. O. Alves, F. J. S. A. Corrêa, and T. F. Ma, "Positive solutions for a quasilinear elliptic equation of Kirchhoff type," Computers \& Mathematics with Applications, vol. 49, no. 1, pp. 85-93, 2005.

[9] B. T. Cheng, "New existence and multiplicity of nontrivial solutions for nonlocal elliptic Kirchhoff type problems," Journal of Mathematical Analysis and Applications, vol. 394, no. 2, pp. 488-495, 2012.

[10] Z. Zhang and K. Perera, "Sign changing solutions of Kirchhoff type problems via invariant sets of descent flow," Journal of Mathematical Analysis and Applications, vol. 317, no. 2, pp. 456463, 2006.

[11] Q.-G. Zhang, H.-R. Sun, and J. J. Nieto, "Positive solution for a superlinear Kirchhoff type problem with a parameter," Nonlinear Analysis: Theory, Methods \& Applications, vol. 95, pp. 333-338, 2014. 
[12] A. M. Mao and Z. T. Zhang, "Sign-changing and multiple solutions of Kirchhoff type problems without the P.S. condition," Nonlinear Analysis: Theory, Methods \& Applications, vol. 70, no. 3, pp. 1275-1287, 2009.

[13] J.-J. Sun and C.-L. Tang, "Existence and multiplicity of solutions for Kirchhoff type equations," Nonlinear Analysis: Theory, Methods \& Applications, vol. 74, no. 4, pp. 1212-1222, 2011.

[14] K. Perera and Z. T. Zhang, "Nontrivial solutions of Kirchhofftype problems via the Yang index," Journal of Differential Equations, vol. 221, no. 1, pp. 246-255, 2006.

[15] C.-Y. Lei, J.-F. Liao, and C.-L. Tang, "Multiple positive solutions for Kirchhoff type of problems with singularity and critical exponents," Journal of Mathematical Analysis and Applications, vol. 421, no. 1, pp. 521-538, 2015.

[16] X. H. Tang and B. T. Cheng, "Ground state sign-changing solutions for Kirchhoff type problems in bounded domains," Journal of Differential Equations, vol. 261, no. 4, pp. 2384-2402, 2016.

[17] L. Zhao and N. Zhang, "Existence of solutions for a higher order Kirchhoff type problem with exponential critical growth," Nonlinear Analysis: Theory, Methods \& Applications, vol. 132, pp. 214-226, 2016.

[18] J.-F. Liao, X.-F. Ke, C.-Y. Lei, and C.-L. Tang, "A uniqueness result for Kirchhoff type problems with singularity," Applied Mathematics Letters, vol. 59, pp. 24-30, 2016.

[19] J. H. Jin and X. Wu, "Infinitely many radial solutions for Kirchhoff-type problems in $\mathbb{R}^{N}$," Journal of Mathematical Analysis and Applications, vol. 369, no. 2, pp. 564-574, 2010.

[20] X. Wu, "Existence of nontrivial solutions and high energy solutions for Schrödinger Kirchhoff-type equations in $R^{N}$," Nonlinear Analysis: Real World Applications, vol. 12, no. 2, pp. 1278-1287, 2011.

[21] J. J. Nie, "Existence and multiplicity of nontrivial solutions for a class of Schrödinger-Kirchhoff-type equations," Journal of Mathematical Analysis and Applications, vol. 417, no. 1, pp. 6579, 2014.

[22] X. M. He and W. M. Zou, "Existence and concentration behavior of positive solutions for a Kirchhoff equation in $\mathrm{R}^{3}$," Journal of Differential Equations, vol. 252, no. 2, pp. 1813-1834, 2012.

[23] J. Wang, L. X. Tian, J. X. Xu, and F. B. Zhang, "Multiplicity and concentration of positive solutions for a Kirchhoff type problem with critical growth," Journal of Differential Equations, vol. 253, no. 7, pp. 2314-2351, 2012.

[24] J. T. Sun and T.-F. Wu, "Ground state solutions for an indefinite Kirchhoff type problem with steep potential well," Journal of Differential Equations, vol. 256, no. 4, pp. 1771-1792, 2014.

[25] G. B. Li and H. Y. Ye, "Existence of positive ground state solutions for the nonlinear Kirchhoff type equations in $\mathbb{R}^{3}$," Journal of Differential Equations, vol. 257, pp. 566-600, 2014.

[26] Y. B. Deng, S. J. Peng, and W. Shuai, "Existence and asymptotic behavior of nodal solutions for the Kirchhoff-type problems in $\mathbb{R}^{3}$," Journal of Functional Analysis, vol. 269, no. 11, pp. 35003527, 2015.

[27] X. Luo and Q. F. Wang, "Existence and asymptotic behavior of high energy normalized solutions for the Kirchhoff type equations in $\mathbb{R}^{3}$," Nonlinear Analysis: Real World Applications, vol. 33, pp. 19-32, 2017.

[28] Q. Xie, S. Ma, and X. Zhang, "Infinitely many bound state solutions of Kirchhoff problem in $\mathbb{R}^{3}$," Nonlinear Analysis: Real World Applications, vol. 29, pp. 80-97, 2016.
[29] Z. S. Liu and S. J. Guo, "Positive solutions for asymptotically linear Schrödinger-Kirchhoff-type equations," Mathematical Methods in the Applied Sciences, vol. 37, no. 4, pp. 571-580, 2014.

[30] Y. Wu and S. B. Liu, "Existence and multiplicity of solutions for asymptotically linear Schrödinger-Kirchhoff equations," Nonlinear Analysis: Real World Applications, vol. 26, pp. 191-198, 2015.

[31] L.-L. Wang and Z.-Q. Han, "Multiple small solutions for Kirchhoff equation with local sublinear nonlinearities," Applied Mathematics Letters, vol. 59, pp. 31-37, 2016.

[32] P. Chen and X. H. Tang, "Existence and multiplicity results for infinitely many solutions for Kirchhoff-type problems in $\mathbb{R}^{N}$," Mathematical Methods in the Applied Sciences, vol. 37, no. 12, pp. 1828-1837, 2014.

[33] L. P. Xu and H. B. Chen, "Nontrivial solutions for Kirchhoff-type problems with a parameter," Journal of Mathematical Analysis and Applications, vol. 433, no. 1, pp. 455-472, 2016.

[34] Y. S. Huang and Z. Liu, "On a class of Kirchhoff type problems," Archiv der Mathematik, vol. 102, no. 2, pp. 127-139, 2014.

[35] Y. Wu, Y. Huang, and Z. Liu, "On a Kirchhoff type problem in $\mathbb{R}^{N}$," Journal of Mathematical Analysis and Applications, vol. 425, no. 1, pp. 548-564, 2015.

[36] Y. H. Li, F. Y. Li, and J. P. Shi, "Existence of a positive solution to Kirchhoff type problems without compactness conditions," Journal of Differential Equations, vol. 253, no. 7, pp. 2285-2294, 2012.

[37] J. Liu, J.-F. Liao, and C.-L. Tang, "Positive solution for the Kirchhoff-type equations involving general subcritical growth," Communications on Pure and Applied Analysis, vol. 15, no. 2, pp. 445-455, 2016.

[38] H. N. Fan and X. C. Liu, "Positive and negative solutions for a class of Kirchhoff type problems on unbounded domain," Nonlinear Analysis: Theory, Methods \& Applications, vol. 114, pp. 186-196, 2015.

[39] H. Y. Ye and F. L. Yin, "The existence of positive solutions to Kirchhoff type equations in $R^{N}$ with asymptotic nonlinearity," Journal of Mathematics Research, vol. 6, pp. 14-23, 2014.

[40] L. Li and J. J. Sun, "Existence and multiplicity of solutions for the Kirchhoff equations," Nonlinear Analysis: Real World Applications, vol. 2, pp. 391-399, 2015.

[41] L. Li and X. Zhong, "Infinitely many small solutions for the Kirchhoff equation with local sublinear nonlinearities," Journal of Mathematical Analysis and Applications, vol. 435, no. 1, pp. 955-967, 2016.

[42] H. B. Chen, H. L. Liu, and L. P. Xu, "Existence and multiplicity of solutions for nonlinear Schrödinger-Kirchhoff-type equations," Journal of the Korean Mathematical Society, vol. 53, no. 1, pp. 201-215, 2016.

[43] C. S. Chen and Q. Zhu, "Existence of positive solutions to p-Kirchhoff-type problem without compactness conditions," Applied Mathematics Letters, vol. 28, pp. 82-87, 2014.

[44] C. S. Chen and Q. Yuan, "Existence of solution to p-Kirchhoff type problem in $R^{N}$ via Nehari manifold," Communications on Pure and Applied Analysis, vol. 1, no. 6, pp. 2289-2303, 2014.

[45] L. Liu and C. Chen, "Study on existence of solutions for $p$ Kirchhoff elliptic equation in $\mathbb{R}^{N}$ with vanishing potential," Journal of Dynamical and Control Systems, vol. 20, no. 4, pp. 575-592, 2014.

[46] X. Y. Chen and G. W. Dai, "Positive solutions for p-Kirchhoff type problems on $\mathbb{R}^{N}$," Mathematical Methods in the Applied Sciences, vol. 38, pp. 2650-2662, 2015. 
[47] H. N. Fan and X. C. Liu, "Multiple positive solutions of degenerate nonlocal problems on unbounded domain," Mathematical Methods in the Applied Sciences, vol. 38, no. 7, pp. 1282-1291, 2015.

[48] Y. Duan and C. L. Tang, "Multiple positive solutions for superlinear Kirchhoff type problems on $\mathbb{R}^{N}$," Electronic Journal of Differential Equations, vol. 2015, no. 316, pp. 1-14, 2015.

[49] J. R. Santos Junior, "The effect of the domain topology on the number of positive solutions of an elliptic Kirchhoff problem," Nonlinear Analysis: Real World Applications, vol. 28, pp. 269283, 2016.

[50] P. H. Rabinowitz, Minimax Methods in Critical Point Theory with Applications to Differential Equations, vol. 65 of CBMS Regional Conference Series in Mathematics, American Mathematical Society, Providence, RI, USA, 1986.

[51] J. Mawhin and M. Willem, Critical Point Theory and Hamiltonian Systems, vol. 74 of Applied Mathematical Sciences, Springer, New York, NY, USA, 1989. 


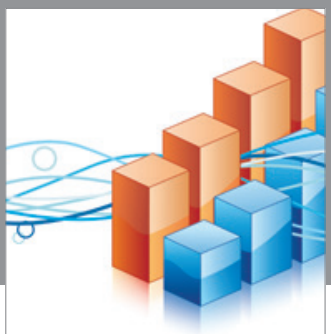

Advances in

Operations Research

vatem alat4

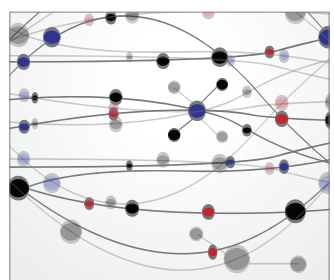

\section{The Scientific} World Journal
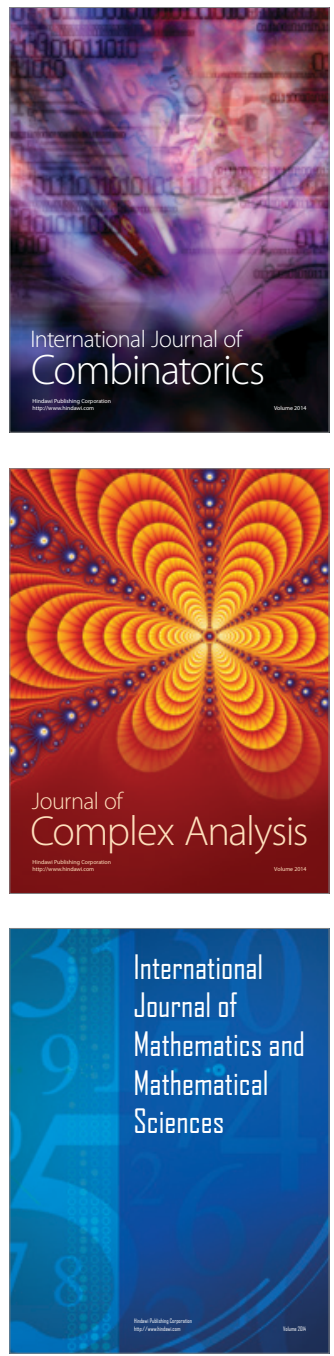
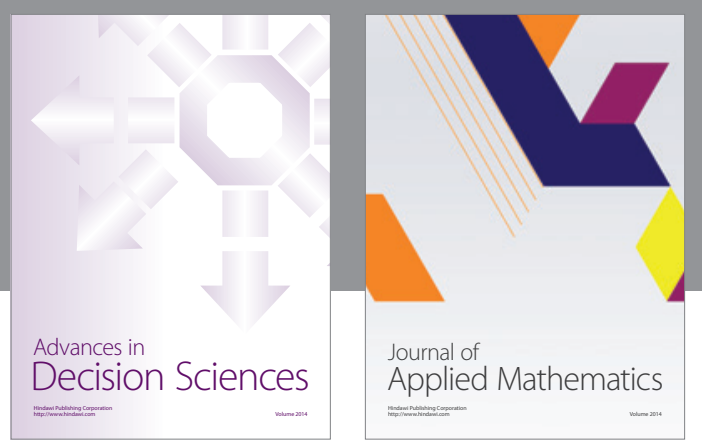

Algebra

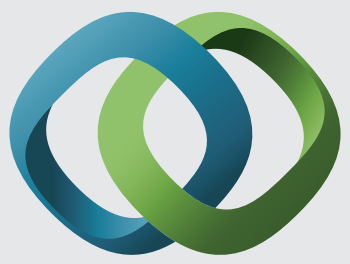

\section{Hindawi}

Submit your manuscripts at

http://www.hindawi.com
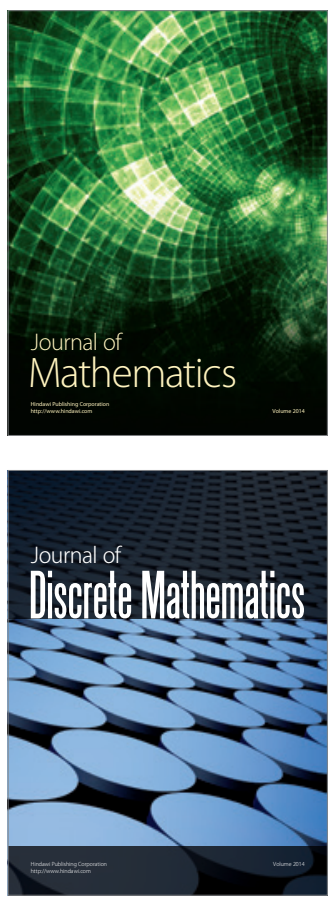

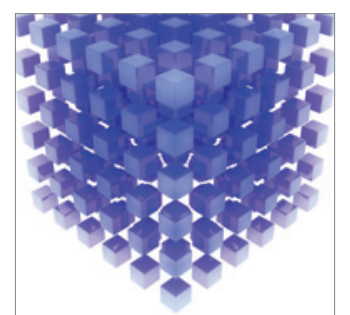

Mathematical Problems in Engineering
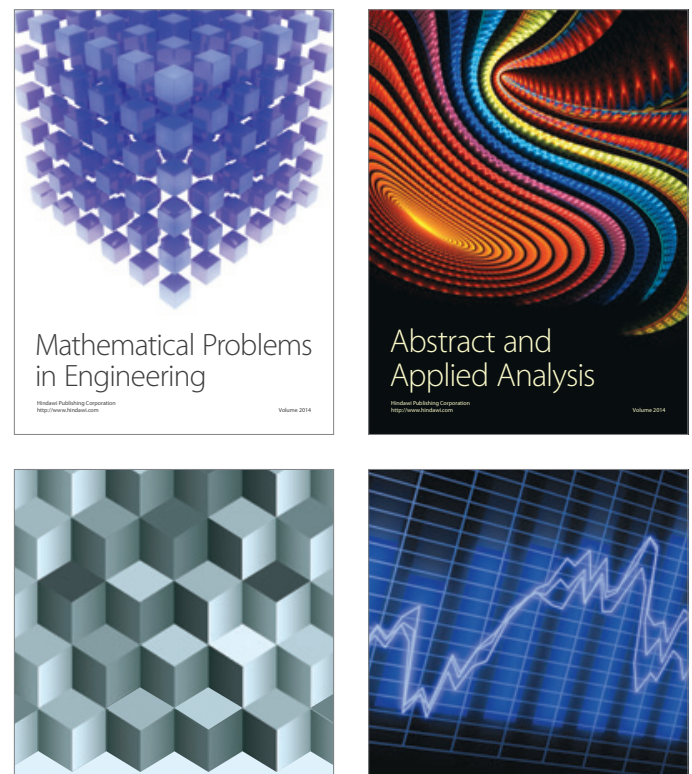

Journal of

Function Spaces

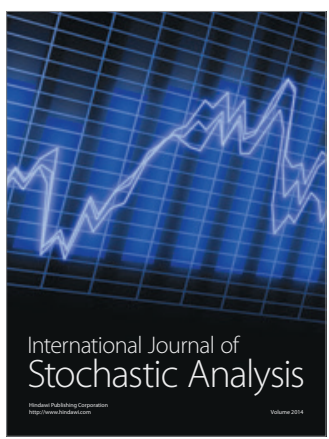

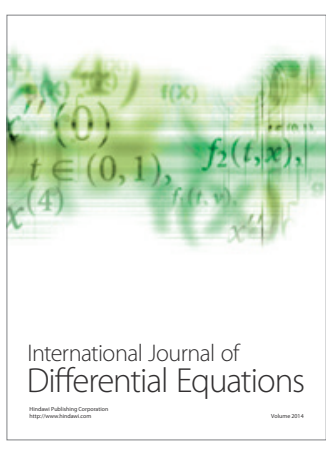
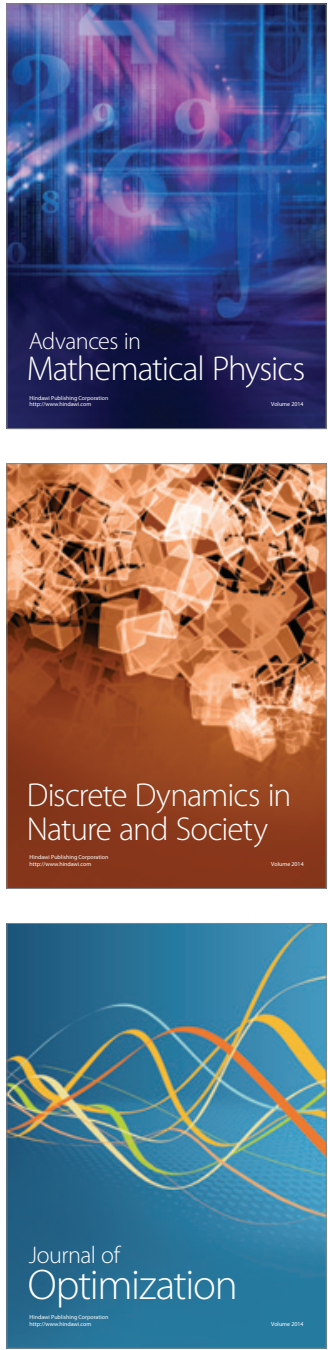